\title{
Correspondence
}

\section{Don't forget the patient}

Day et al's study of thiamine prescribing ${ }^{1}$ was interesting and valuable. The results given in the abstract report only the small positive change in the post-intervention group, rather than reflecting the mixed picture of positive and negative change in the appropriateness of prescribing which are outlined more fully in the body of the paper. It is concerning and disappointing that such a clear and ostensibly easy-to-use flowchart did not produce the degree of change in practice that one might reasonably have hoped for, and still left the significant majority of patients apparently receiving suboptimal treatment.

The authors highlight the role of clinician-dependent factors, such as incomplete history taking on admission, lack of knowledge and disproportionate concern with rare adverse reactions. I would argue that the relative failure of an information-giving intervention to produce real improvements in clinical practice should encourage us to look more deeply at the patient-related factors which may act as barriers to the delivery of 'optimal' treatment.

From my own clinical experience, I would suggest that factors such as patient concordance, cooperativeness and capacity are major determinants of the feasibility of delivering what, on paper, would be best practice. Patients with chronic alcohol misuse not uncommonly have comorbid psychiatric conditions or personality styles which affect their adherence to the relatively unpleasant treatments of cannulation and intramuscular injection. Acute confusion, noted in around a third of the sample in Day et al's study, would often impair the capacity to consent to treatment. The risks, to staff and the patient alike, of attempting to administer thiamine parenterally to an uncooperative individual are considerable, and must be evaluated in any best-interests decision-making process. Such patient-related factors may explain the preference among treating professionals to take the route of oral medication despite advice to the contrary, particularly in less clearly defined cases.

I look forward to seeing further exploration of factors that bear influence on the delivery of treatment in future studies.

1 Day E, Callaghan R, Kuruvilla T, George S, Webb K, Bentham P. Pharmacy-based intervention in Wernicke's encephalopathy. Psychiatrist 2010; 34: 234-8.

Ruth V. Reed, Specialty Registrar in Child and Adolescent Psychiatry, Oxfordshire and Buckinghamshire Mental Health NHS Foundation Trust, Abingdon, email: ruthvreed@gmail.com

doi: $10.1192 /$ pb.34.9.400

\section{Care pathways mislabel and mislead}

Care pathways originated in the North East, Yorkshire and Humber regions and I understand why the current 21 clusters have been developed. There is a need to measure what psychiatric services do and develop objective ways of assessing outcome rather than process. ${ }^{1}$ The problem is that this newly imposed system does not achieve these objectives. Its main measure of outcome, the Health of the Nation Outcome Scales (HoNOS), was developed in the 1990s at a time when the focus was on psychotic illnesses. At the time there were concerns about the instrument's sensitivity to change and ability to measure outcome. ${ }^{2,3}$ In 2010, the focus has broadened to include statistically more significant health challenges such as stress disorders, substance misuse, somatoform disorders, personality disorder, anxiety and depression. HoNOS remains a helpful tool in rehabilitation services and forensic settings, but its applicability to general and community psychiatry is limited. Using it on a day-to-day basis, as I have been instructed to, it smacks of a system that is out of date and that simply does not address the heart of the matter.

For example, if somebody has psychotic experiences as a result of drinking alcohol, the computerised system will allocate that individual to a psychotic pathway even though it is clear that alcohol had a causal role. There is only one care pathway for substance misuse despite the variations in substances, legality and levels of addiction and yet there are eight pathways for psychosis. There is no appropriate care pathway for eating disorders, nor is there any specific enquiry at any point about whether a person is losing weight.

It does not surprise me that anecdotal findings suggest that many people referred to general psychiatry are categorised into the common and mild pathways, 1 and 2. This is a problem with the unbalanced nature of the assessment tool rather than the referral process. It alarms me when I hear commissioners and senior mental health trust managers suggesting that psychiatric services should not see such patients. This may lead the local communities that we serve to perceive us as increasingly irrelevant.

Care pathways are a bureaucratic procedure. It is labour intensive and competes with other documentation processes for time spent in direct face-to-face contact with patients. In my view, the process has the ability to mislead clinicians, managers and the general public. It also has the power to offend some service users by labelling their distressing conditions as, for example, 'common mental health problems (low severity)'. As a professional body, I think we should ask the question, is this a good enough measure to underpin payment by results?

1 Department of Health. Practical Guide to Preparing for Mental Health Payment by Results. Department of Health, 2009.

2 Trauer T, Callaly T, Hantz P, Little J, Shields R, Smith J. Health of the Nation Outcome Scales. Results of the Victorian field trial. Br J Psychiatry 1999; 174: 380-8.

3 Bebbington P, Brugha T, Hill T, Marsden L, Window S. Validation of the Health of the Nation Outcome Scales. Br J Psychiatry 1999; 174: 389-94.

Martin A. Gee, Consultant Adult Psychiatrist, North Staffordshire Combined Healthcare NHS Trust, email: m.a.gee@doctors.org.uk

doi: 10.1192/pb.34.9.400a

A 'meaning-centred approach' to patient consultation is the same as spirituality and psychiatry

I commend Paul Wallang's excellent piece, ${ }^{1}$ which is as brilliant as it is relevant. I cannot agree more with the contents of his 